\title{
Compromiso e inteligencia emocional en mediadores del Poder Judicial de Oaxaca, México*
}

\section{Commitment and Emotional Intelligence in Mediators of the Judicial Power of Oaxaca, Mexico}

\section{Carmen María Salvador Ferrer* \\ Universidad de Almería, España}

Eric García-López

Universidad Autónoma Benito Juárez,

Oaxaca, México

Recibido: 25 de enero de 2010

Revisado: 1 de marzo de 2010

Aceptado: 15 de abril de 2010

\section{Resumen}

Este trabajo es un estudio transversal descriptivo, cuyo interés es conocer el papel que desempeñan el compromiso organizacional y la inteligencia emocional en la labor de los mediadores mexicanos. Para tal finalidad, se utilizó una muestra de 38 mediadores del Poder Judicial de Oaxaca. Además, para realizar el análisis de la situación, a través de ecuaciones estructurales, nos centraremos en el estudio de diferentes constructos, tales como: el compromiso, la inteligencia emocional, el sexo y la edad. Así, los resultados de esta investigación indican el impacto que estas variables tienen en el comportamiento del mediador. Además, encontramos que, según la edad $y$ el sexo de los participantes, existen notables diferencias en los componentes del compromiso e inteligencia emocional.

Palabras clave: ecuaciones estructurales, mediadores, sexo, edad, inteligencia emocional, compromiso organizacional.

\footnotetext{
Este artículo forma parte de los trabajos de investigación realizados durante la estancia posdoctoral, financiada por el Consejo Nacional de Ciencia y Tecnología de México (CONACYT)"

Correspondencia: Carmen María Salvador Ferrer. Universidad de Almería (España). Facultad de Psicología. Área de Psicología Social. Carretera de Sacramento. La Cañada de San Urbano, s/n. C.P.04120. Almería. Tlf.:950014047. Correo electrónico: cmsalva@ual.es. Eric García-López. Universidad Autónoma "Benito Juárez" de Oaxaca, México. Torre de Rectoría. Av. Universidad s/n, Ex-Hacienda de Cinco Señores. Oaxaca, México. C.P. 68120. Correo electrónico: ericgarcia@psicologiajuridica.org
} 


\section{Abstract}

This work is a descriptive traverse study that deposits the interest in knowing the paper that you/ they carry out the organizational commitment and the emotional intelligence in the work of the Mexican mediators. For such a purpose, a sample of 38 mediators of the Judicial Power of Oaxaca is used. Also, to carry out the analysis of the situation, through structural equations, we will center ourselves in the study of different aspects, such as, the commitment, the emotional intelligence, the sex and the age. This way, the results of this investigation indicate the impact that these variables have in the mediator's behavior. Also, we find that, according to the age and the sex of the participants, notables differences exist in the components of the commitment and emotional intelligence.

Key words: structural equations, mediators, emotional intelligence, age, sex, commitment.

\section{Introducción}

El objetivo establecido en el presente trabajo se centra en analizar, a través de ecuaciones estructurales, cómo influyen distintas variables psicológicas (inteligencia emocional y el compromiso) en determinados rasgos de personalidad (sexo y edad) de los mediadores del Poder Judicial de Oaxaca. En este sentido, con los resultados que se desprendan de este estudio, podremos conocer qué aspectos resultan fundamentales en el perfil ideal del mediador mexicano.

\section{Compromiso organizacional}

Uno de los problemas que más ha preocupado a los teóricos de las organizaciones ha sido, y sigue siendo, conseguir el compromiso de los individuos que forman parte de la institución para que concentren todos sus esfuerzos en la consecución de metas corporativas y, por tanto, logren los objetivos (Guest, 1995; Salvador, 2005; Salvador \& Mañas, 2009). Se convierte así el compromiso en una variable fundamental en la eficiencia, productividad y efectividad institucional (Lyons, Duxbury \& Higgins, 2006; Meyer \& Allen, 1997; Meyer, Stanley, Herscovithch \& Topolnytky, 2002; Morgan \& Hunt, 1994; Salvador, 2005).

El interés por este término ha llevado a que se impulsen numerosos trabajos en una variedad de contextos y, además, se ha aplicado a una diversidad de personas en diferentes situaciones. En este sentido, podemos encontrar estudios no sólo en el ámbito organizacional y marketing (Allen \& Meyer, 1990; Meyer \& Allen, 1984), sino también en otros campos como la Psicología (Becker, 1960; Salancik, 1977) y Economía (Cook \& Emerson, 1978). Como consecuencia, han surgido numerosas definiciones, entre otras, resaltamos la ofrecida por Porter \& Lawler (1965), quienes entienden el compromiso como el deseo de realizar elevados esfuerzos por el bien de la institución, el anhelo de permanecer en la misma y aceptar sus principales objetivos y valores.

En otro ángulo, Kanter (1968) mantiene, desde una perspectiva ideal, que el compromiso se debe cimentar en unas bases algo más complejas que un simple intercambio de material. Por su parte, Buchanan (1974) ve al individuo comprometido como un miembro de la institución, lo cual genera un sentimiento de apego hacia los objetivos y valores organizacionales. Siguiendo a Etzioni (1975), el compromiso es la implicación positiva del individuo en la institución. Con el mismo sentido, Franklin (1975) fundamenta este concepto en el deseo de acatar las normas de la organización y permanecer en la misma.

Por su parte, Cotton (1993) entiende el compromiso como un proceso participativo, resultado de combinar información, influencia incentivos, donde se usan todas las capacidades personales para estimular el apego de los empleados hacia el éxito institucional. Robbins (1999) señala que el compromiso organizacional es uno de los tres 
tipos de actitudes que una persona tiene relacionados con su trabajo y lo define como un estado en el cual un empleado se identifica con una organización particular y con sus metas y desea mantenerse en ella como uno de sus miembros.

Pese a que se han propuesto múltiples definiciones del compromiso, todas coinciden en señalar que se trata de una vinculación que establece el individuo con la organización. Este hecho conduce a que el estudio del compromiso organizacional haya adoptado tres perspectivas diferentes, en concreto: la Teoría del intercambio; la perspectiva psicológica y, finalmente, la perspectiva de atribución.

Desde la perspectiva de intercambio social, Becker (1960) definió el compromiso organizacional como el vínculo que establece el individuo con su organización, fruto de las pequeñas inversiones (side-best) realizadas a lo largo del tiempo. Según esto, la persona continúa en la organización porque cambiar su situación supondría sacrificar las inversiones realizadas.

Desde la perspectiva psicológica, el compromiso con la organización se entiende como la fuerza relativa a la identificación individual e implicación con una organización en particular, y puede ser caracterizado por un fuerte deseo de permanecer como miembro de una organización en particular, un acuerdo de mantener altos niveles de esfuerzo en beneficio de la organización y una creencia definitiva y aceptación de los valores y metas de la organización, que resulta de la orientación individual hacia la organización como un fin en sí mismo (Mowday, Steers \& Poter, 1979), ya que las personas comprometidas manifiestan cierta intensidad en la unión psicológica (emocional) con la organización (O'Reilly \& Chatman, 1986). En este sentido, la formación del compromiso psicológico depende del intercambio que mantiene el empleado con la organización, puesto que la persona espera recibir, entre otros aspectos, determinadas recompensas psicológicas como obtener nuevos conocimientos, reconocimiento de un grupo de trabajo (Mathieu \& Zajac, 1990).
La tercera corriente es la perspectiva de atribución que define el compromiso como una obligación que el individuo adquiere como resultado de realizar ciertos actos que son voluntarios, explícitos e irrevocables (Reichers, 1986). Esta dimensión está más vinculada a la dimensión de "ética del trabajo" propuesta por Morrow (1983). Dicha ética puede ser definida como una responsabilidad que nace del interior del individuo y que impulsa a hacer el mayor esfuerzo posible por realizar un buen trabajo (Varona, 1993).

Desde otro ángulo, más recientemente, Meyer, Allen y Smith (1993) proponen una conceptualización del compromiso organizacional dividido en tres componentes: afectivo, de continuidad (calculado) y normativo. De esta manera, y según estos autores, la naturaleza del compromiso es, respectivamente, el deseo, la necesidad o el deber permanecer en la organización. Para facilitar la interpretación de los términos, mostramos a continuación su delimitación conceptual de los mismos.

El compromiso afectivo es el más ampliamente estudiado, se refiere a los lazos emocionales que las personas forjan con la organización. En este sentido, refleja el apego emocional al percibir la satisfacción de necesidades (especialmente las psicológicas) y expectativas, disfrutando de su permanencia en la organización. Los trabajadores con este tipo de compromiso se sienten orgullosos de pertenecer a la organización. Aún más, Mowday, Steers y Porter (1979) lo definen como la fuerza de la identificación de un individuo con una organización en particular y de su participación en la misma. Conceptualmente, puede ser caracterizado por al menos tres factores: (a) una fuerte convicción y aceptación de los objetivos y valores de la organización; (b) la disposición a ejercer un esfuerzo considerable en beneficio de la organización y, finalmente, (c) el fuerte deseo de permanecer como miembro de la organización.

El compromiso de continuidad es la segunda forma de compromiso más investigada. Ésta señala el reconocimiento de la persona con respecto a los costos (financieros, físicos y psicológicos) y las pocas oportunidades de encontrar otro empleo, si la persona decidiera renunciar a la organiza- 
ción. Se construye a partir de la teoría del "sidebet" de Becker de la década de los sesenta. Los "side -bets" son las inversiones valoradas por el individuo que serían pérdidas si éste dejara la organización (Cohen \& Lowenberg, 1990). Este tipo de compromiso es una formulación más reciente del compromiso calculado (Morrow, 1983), incorporando la idea de facilidad de movimiento del trabajador de una organización a otra.

Por último, el compromiso normativo ha sido el menos analizado empíricamente. Es aquél que encuentra la creencia en la lealtad de la organización, en un sentido moral, de alguna manera como pago, quizá por recibir ciertas prestaciones. En este tipo de compromiso se desarrolla un fuerte sentimiento de permanecer en la institución, como efecto de experimentar una sensación de deuda hacia la organización por haberle dado la oportunidad o recompensa que fue valorada por el trabajador. Este sentimiento de lealtad del individuo con la organización puede deberse, también, a presiones de tipo cultural y familiar (Morrow, 1983).

Siguiendo las tres vertientes del compromiso, expuestas anteriormente, consideramos que el compromiso afectivo es una de las dimensiones más relevantes (Salvador, 2005; 2007, 2008a y b; 2009) puesto que, para lograr un mayor compromiso la organización debe considerar a los empleados como personas y brindarle apoyo en todo momento, especialmente en situaciones de emergencia. En este sentido, una persona puede estar obligada formalmente a desempeñar una labor dentro de una organización, pero si no se liga afectivamente a la misma, puede trabajar incómodamente e incluso llegar a sentir desprecio hacia la organización. Como consecuencia de todo esto, presumiblemente, podríamos esperar un rendimiento menor en el trabajo.

De igual forma, podrían desprenderse otras posibles consecuencias negativas, tales como las que relatamos a continuación: estrés, conflictos obrero-patronales, ausentismo, abandono de la organización, etc. Así, en el caso de que no se fragüe el vínculo afectivo entre el individuo y la organización, podríamos pronosticar que los cos- tes, tanto para la institución como para el individuo, se vean afectados, dicho con otras palabras, venimos a reflejar que sin esta relación afectiva el esfuerzo para el individuo será mayor y el coste económico para la empresa incrementará como consecuencia de los gastos asociados al mismo (por ejemplo, las bajas laborales). En este sentido, de acuerdo con Eisenberger, Hungtington, Hutchinson \& Sowa (1986), si las personas perciben que la organización se interesa por ellas, procura su bienestar y les brinda ayuda ante problemas personales cuando es necesario, las consecuencias son muy favorables para la organización en cuanto al desempeño y permanencia.

En una línea bastante próxima encontramos los planteamientos propuestos por Arciniega (2002), quien explica que todo colaborador experimenta las tres dimensiones, aunque, desde luego, con distinto énfasis. En un intento de ser más precisos, este autor utiliza la analogía de las olimpiadas para explicar el papel que desempeña cada una de las vertientes que componen el compromiso. En este sentido, el resultado de graduación sería: oro para el afectivo; plata para el normativo y bronce para el continuo. Desde la perspectiva de este autor, los resultados se entienden si consideramos al compromiso afectivo como el colaborador que manifiesta actitudes, tales como, el marcado orgullo de pertenencia hacia la empresa. Este hecho hace que el individuo se emocione al decir que trabaja para la institución o, incluso, habla mucho de ella en reuniones con amigos y familiares.

Resumiendo, la clave para conseguir el compromiso organizativo y, por tanto, para sentar las bases de un servicio perfecto, es la implicación de todas las personas. Dicha implicación se refleja en la aceptación y creencia de las metas y valores organizacionales, en los esfuerzos realizados por sus integrantes (empleados y clientes) en nombre de la institución y en el deseo de seguir siendo miembros de la misma. De esta forma, únicamente las instituciones integradas por personas comprometidas con sus objetivos, con unos propósitos comunes y con responsabilidad mutua y compartida podrán dar ese salto cualitativo que supone el tránsito hacia formas estructurales más adaptadas y flexibles (Salvador, 2008b). 


\section{Inteligencia emocional}

En la última década la investigación sobre regulación y manejo de las emociones ha experimentado un gran interés (Rego \& Fernández, 2005; Salvador, 2008 a y b, 2009; Salvador \& Morales, 2009). A grandes rasgos, podemos definir la inteligencia emocional como una forma de interactuar con el mundo que tiene muy en cuenta los sentimientos, y engloba habilidades, tales como el control de los impulsos, la autoconciencia, la motivación, el entusiasmo, la perseverancia, la empatía, la agilidad mental, entre otras. Todos estos rasgos de personalidad configuran el carácter como la autodisciplina, la compasión o el altruismo, que resultan indispensables para una buena y creativa adaptación social. Grosso modo, las personas con habilidades emocionales bien desarrolladas también tienen más probabilidades de sentirse satisfechas y ser eficaces en su vida.

Salovey \& Mayer (1990) consideran que la inteligencia emocional se apoya en cinco aspectos cruciales. En primer lugar, conocer las propias emociones, esto es, la conciencia de uno mismo y la capacidad de controlar sentimientos (auto-conocimiento). En segundo lugar, manejar las emociones, entendida como la capacidad de manejar sentimientos para que sean adecuados, este aspecto se basa en la conciencia de uno mismo (auto-control). En tercer lugar, la propia motivación, es decir, la capacidad de ordenar las emociones al servicio de un objetivo esencial (auto-motivación). En cuarto lugar, reconocer las emociones de los demás, se trata de la empatía (autoconciencia de las emociones de los otros). Por último, en quinto lugar, manejar las relaciones, entendida como la capacidad de manejar las emociones de los demás $\mathrm{y}$, también, de uno mismo.

En una línea similar, Goleman $(1998,2001)$ y Salvador (2009) consideran que para tener inteligencia emocional es preciso disponer de inteligencias múltiples. En primer lugar, la Inteligencia Personal, la cual, a su vez, está compuesta por una serie de competencias que determinan el modo en que nos relacionamos con nosotros mismos. Siendo más precisos, comprende tres componen- tes: (a) conciencia en uno mismo o capacidad de reconocer y entender en uno mismo las propias fortalezas, debilidades, estados de ánimo, emociones e impulsos, así como el efecto que éstos tienen sobre los demás y sobre el trabajo. Esta competencia se manifiesta en personas con habilidades para juzgarse a sí mismas de forma realista, que son conscientes de sus propias limitaciones y admiten con sinceridad sus errores, que son sensibles al aprendizaje y que poseen un alto grado de auto-confianza; (b) autorregulación o control de sí mismo o habilidad de controlar nuestras propias emociones e impulsos para adecuarlos a un objetivo, de responsabilizarse de los propios actos, de pensar antes de actuar y de evitar los juicios prematuros. Las personas que poseen esta competencia son sinceras e íntegras, controlan el estrés y la ansiedad y, además, se muestran flexibles ante los cambios o las nuevas ideas; y, finalmente, (c) auto-motivación o habilidad de estar en un estado de continua búsqueda y persistencia en la consecución de los objetivos, haciendo frente a los problemas y encontrando soluciones. Esta competencia se manifiesta en las personas que muestran un gran entusiasmo por su trabajo y por el logro de las metas por encima de la simple recompensa económica, con un alto grado de iniciativa y compromiso, y con gran capacidad optimista en la consecución de sus objetivos.

En segundo lugar, la Inteligencia Interpersonal, la cual incluye otras competencias que determinan el modo en que nos relacionamos con los demás. Siendo más precisos, resalta: (a) empatía o habilidad para entender las necesidades, los sentimientos y los problemas de los demás, poniéndose en su lugar y respondiendo correctamente a sus reacciones emocionales; $y$, finalmente, (b) habilidades sociales, entendida como el talento en el manejo de las relaciones con los demás, o sea, en saber persuadir e influenciar a los demás.

Asumiendo los planteamientos teóricos expuestos previamente, el principal propósito de la presente investigación consiste en analizar el papel del compromiso y de la inteligencia emocional en la personalidad de los mediadores del Poder Judicial de Oaxaca. Para ello, las hipótesis de partida que orientan este estudio son las siguientes: 
- Hipótesis 1. Los tres aspectos del compromiso (afectivo, normativo y calculado) ejercerán notables influencias en el género de los participantes, siendo la vertiente normativa la que desempeñará un papel central.

- Hipótesis 2. Los tres componentes del compromiso (afectivo, normativo y calculado) influirán de manera significativa en la edad de los participantes, mostrándose puntuaciones superiores en la vertiente afectiva.

- Hipótesis 3. Los tres componentes de la inteligencia emocional (atención y percepción emocional, claridad emocional y manejo de las emociones) presentan notables repercusiones en la edad de la muestra, siendo ligeramente superior el manejo de las emociones.

- Hipótesis 4. Los componentes de la inteligencia emocional (atención y percepción emocional, claridad emocional y manejo de las emociones) se relacionarán fuertemente con el sexo, mostrándose elevada la relación entre atención y percepción.

- Hipótesis 5. Existe una relación estadísticamente significativa y negativa entre el compromiso afectivo y la atención y percepción emocional.

- Hipótesis 6. Existe una fuerte relación, es decir, estadísticamente significativa y positiva, entre el compromiso afectivo y la claridad de los sentimientos.

\section{Método}

\section{Muestra}

La investigación de carácter descriptivo transversal, realizada en México, cuenta con 38 participantes del Poder Judicial de Oaxaca, de los cuales un $51 \%$ son hombres y el $49 \%$ restante mujeres. Las edades se encuentran comprendidas entre los 24 y 73 años, siendo la media de la edad de 38.15 (desviación típica de 12.34). Además, según el objetivo establecido a lo largo del presente trabajo, consideramos apropiado sobresaltar una característica relacionada con la unidad muestral, en concreto, el hecho de que todos los participantes intervinieran de manera voluntaria. Por último, otro aspecto que merece la pena destacar es el hecho de que más de la mitad de los participantes mostraran deseo de permanecer el resto de la vida profesional en dicha organización (media de 3.89, desviación típica de 1.22).

\section{Instrumentos}

A continuación, se detallan las características de las escalas y las propiedades psicométricas más relevantes de los instrumentos de evaluación empleados (Salvador, 2005, 2008b; Salvador \& Morales, 2009):

Compromiso organizacional. La elaboración del instrumento se ciñó a las recomendaciones ofrecidas por Churchill (1979) para la construcción de escalas. Este autor recoge la complejidad de dimensiones posibles (percepciones, sentimientos, actitudes y conductas) y, además, entre otros aspectos, deja entrever que es más importante disponer de una escala reducida, fiable y válida que contar con un cuestionario compuesto por una gran cantidad de preguntas. En este sentido, en el presente trabajo analizamos el compromiso con una escala compuesta por seis ítems, similares a los empleados por Meyer \& Allen (1984) y Salvador (2005). Concretamente, seguiremos la adaptación al castellano ofrecida por Frutos y cols. (1998) y Salvador (2005), de donde se seleccionaron dos ítems para cada vertiente (afectiva, normativa y calculada). El formato de respuesta de la escala es tipo Likert de siete puntos (Peter, 1979), indicando el valor 1 como "desacuerdo absoluto" con lo que se refleja en la pregunta y el valor 7 todo lo contrario, siendo esta escala multi-ítem la modalidad más utilizada para analizar el compromiso (Salvador, 2005, 2008 a y b). Como primera medida de calidad del instrumento se recurrió al análisis de consistencia (Churchill, 1979). En nuestro caso el coeficiente alfa general de Cronbach es de .693 .

Inteligencia emocional. Este instrumento engloba todos los ítems utilizados para evaluar la inteligencia emocional, procedentes de las dos herramientas tradicionales en su versión en castellano (TMMS y SWLS) aplicadas en el trabajo de Salvador (2008a). En este sentido, el total de pregun- 
tas de esta escala asciende a 53, de las cuales 24 corresponden a la primera y el resto a la segunda. En ambos casos, se evalúa el grado de acuerdo sobre una escala tipo Likert de 5 puntos, indicando el valor 1 "un desacuerdo absoluto" y el valor 5 todo lo contrario. El coeficiente de fiabilidad, en este caso, es de .869 .

\section{Procedimiento}

El presente estudio se integra en el marco de una investigación internacional realizada en el Poder Judicial de Oaxaca (México), en colaboración con la Universidad de Almería. La implantación se llevó a cabo por un grupo de investigadores, presentada en una única sesión cuya duración ascendía a unos 40 minutos en promedio. Considérese que las instrucciones que se ofrecían a los respondentes aseguraban la absoluta confidencialidad y anonimato. Finalmente, los datos fueron analizados con el paquete estadístico Amos $\mathbf{1 7 . 0}$ que permite realizar ecuaciones estructurales (Path diagram).

\section{Resultados}

El hecho de que este trabajo se encuentre en la misma dirección que otros artículos previos elaborados por la autora (Salvador, 2005, 2008a y b; Salvador \& Morales, 2009), justifica que se utilicen las categorías que en las investigaciones citadas inicialmente se encuentran validadas a nivel estructural. Pese a todo, recordaremos, de manera abreviada, que en dichos trabajos cuando se realiza un análisis factorial del compromiso son tres los aspectos que resaltan (afectivo, normativo y de continuidad), mientras que, en este trabajo, en la inteligencia emocional se desprenden un total de tres factores (atención y percepción emocional, claridad en los sentimientos y manejo de las emociones), nótese que estos resultados no coinciden íntegramente los datos obtenidos en otros estudios realizados por la autora, donde se encontraban siempre cuatro categorías (claridad, atención de emociones, manejo de información y utilización de las emociones), véase Salvador, 2008a (estudio realizado en España) y Salvador \& Morales, 2009 (trabajo llevado a cabo en México).
Una vez realizada esta apreciación continuamos con el objetivo del presente estudio, con el cual orientaremos los análisis estadísticos. En concreto, utilizaremos análisis estructurales para averiguar si los componentes del compromiso influyen en el sexo (Figura 1) y, también, en la edad de los participantes (Figura 2).

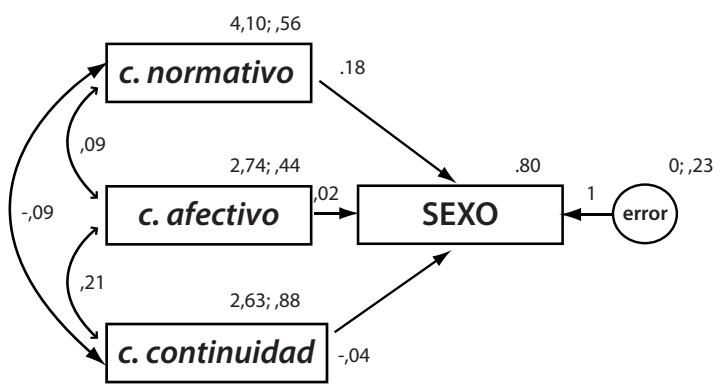

Figura 1. Relación de los componentes del compromiso con el sexo.

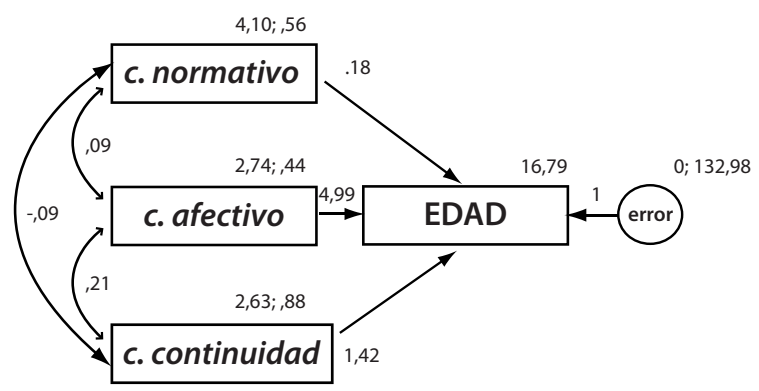

Figura 2. Relación de los componentes del compromiso con la edad.

En lo que respecta a la Figura 1, quisiéramos destacar, en primer lugar, las estrechas relaciones que se desprenden en los tres componentes del compromiso, no siendo el signo el mismo en todos los casos. En concreto, descubrimos que existe una relación negativa entre el compromiso de continuidad y el normativo ( $r=-.09)$, siendo el resto de casos positivos $y$, ligeramente, superior en la asociación compromiso afectivo de continuidad $(r=.21)$. En segundo lugar, resulta necesario distinguir el notable impacto que ejerce el compromiso normativo en el sexo $(r=.18)$.

Cuando nos detenemos en el análisis de los componentes del compromiso en la variable edad 
(Figura 2), descubrimos, en primer lugar, que las intrarelaciones entre los componentes del compromiso tienen el mismo peso y signo, no sucediendo lo mismo en las intercorrelaciones con la edad. En este caso, apreciamos el notable impacto que tienen las tres dimensiones del compromiso, siendo notablemente superior el afectivo $(r=4.99)$.

A modo de síntesis, según los hallazgos obtenidos (figuras 1 y 2), convendría distinguir que los elementos que componen el compromiso influyen tanto en la edad como el sexo de los participantes. No obstante, resulta necesario subrayar las diferencias existentes entre ambas variables. Siendo un poco más precisos, podríamos decir que en el sexo el aspecto que ejerce una mayor influencia es el normativo, mientras que en la edad es el afectivo.

En otro ángulo, cuando nos detenemos en el análisis de los componentes de la inteligencia emocional en el sexo (Figura 3), observamos que los tres elementos integrantes de la inteligencia emocional se relacionan entre sí positivamente, siendo, ligeramente, superior la relación entre atención y percepción y claridad en los sentimientos ( $r=.32)$. Aún más, en la vinculación entre los componentes de la inteligencia y la variable sexo, descubrimos que existe una relación negativa entre el manejo de emociones y el sexo ( $r=-.24)$, siendo positiva las otras dos restantes.

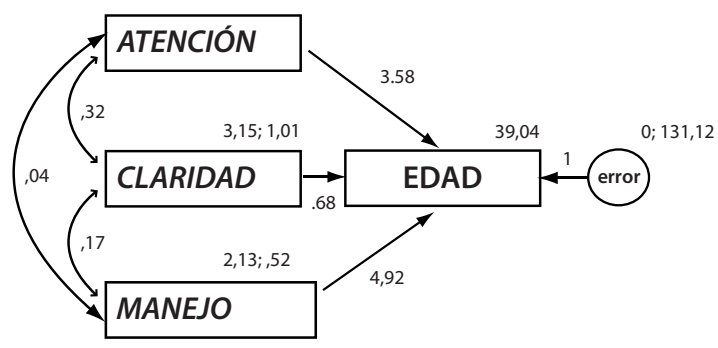

Figura 3. Relación de los componentes la inteligencia emocional con el sexo.
Por último, cuando nos detenemos en estudiar cómo influyen los componentes de la inteligencia emocional en la edad de los participantes (Figura 4), descubrimos que las intracorrelaciones entre los aspectos de la inteligencia son idénticas a las obtenidas anteriormente. Además, cabe señalar que la relación más estrecha con la edad surge en el componente atención y percepción $(r=.68)$.

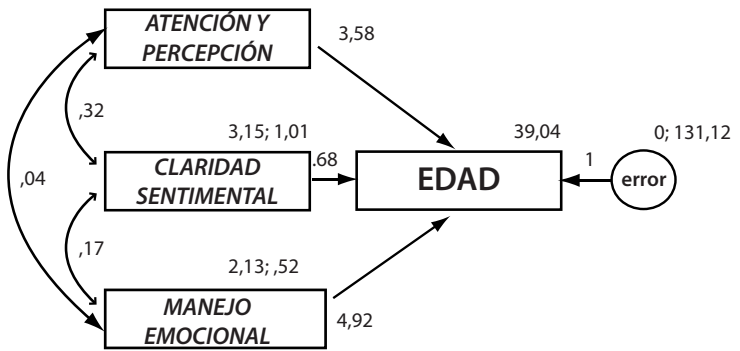

Figura 4. Relación de los componentes la inteligencia emocional con la edad.

En este sentido, a la vista de los resultados obtenidos en ambas figuras ( 3 y 4 ), podríamos decir que los componentes de la inteligencia emocional influyen en la edad y el sexo del perfil de los mediadores mexicanos. Otro aspecto a destacar es la fuerte vinculación que surge entre la atención y percepción y las variables de personalidad (edad y sexo $-r=.18, r=3.59$, respectivamente).

Finalmente, nos detendremos en estudiar el tipo de relación que se produce entre todos los componentes del compromiso organizacional y los aspectos integrantes de la inteligencia emocional (Figura 5). En concreto, descubrimos que el compromiso afectivo se relaciona significativamente y positivamente con los tres aspectos de la inteligencia emocional, siendo la asociación con manejo de las emociones la vertiente más valorada $(r=.25)$. En el lado opuesto, encontramos la categoría de atención y percepción de las emociones $(r=.07)$. 


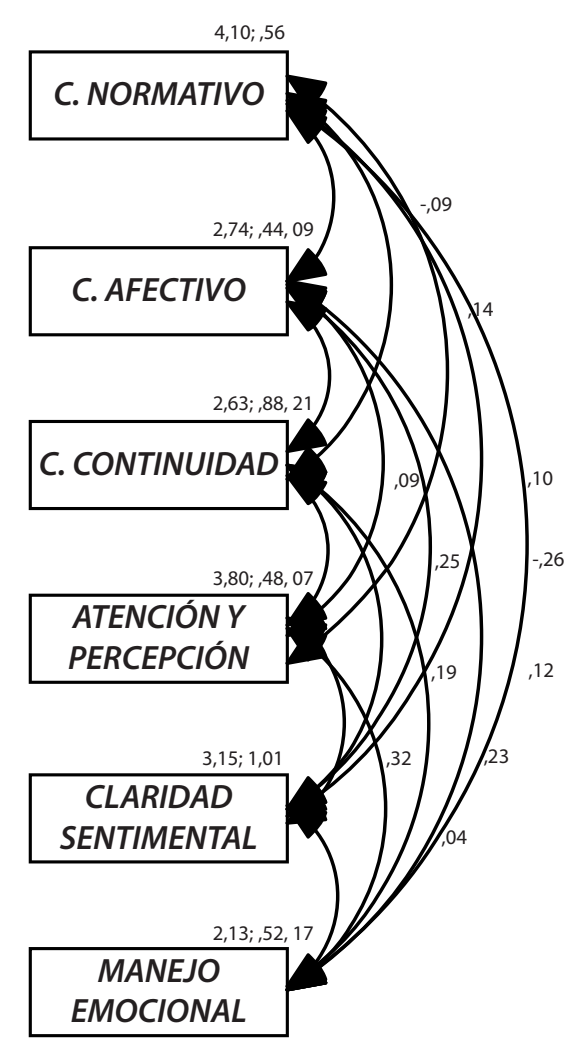

Figura 5. Relación de los componentes del compromiso con la inteligencia emocional.

En el caso del compromiso calculado, quisiéramos destacar que existen relaciones positivas y significativas con todos los elementos de la inteligencia emocional. Siendo un poco más precisos, apreciamos que el mayor impacto se produce con el manejo de las emociones (r0.32), seguido de atención y percepción emocional $(r=.07)$ y, por último, manejo de las emociones $(r=.04)$.

Cuando nos detenemos en el análisis del compromiso afectivo apreciamos que existe una relación positiva y significativa con claridad de los sentimientos $(r=.25)$. Contrariamente, cuando nos centramos en la relación de este término con las dos vertientes restantes (manejo de emociones y atención y percepción emocional), observamos una relación negativa y significativa $(r=.-26 ; r=-$ .09 , respectivamente).

Sintetizando los aspectos más destacables extraídos de la relación entre los aspectos del compro- miso y los elementos de la inteligencia emocional (Figura 5), quisiéramos destacar la existencia de una relación negativa entre el compromiso normativo y manejo de las emociones $(r=-.26)$. En lo que respecta a las relaciones estadísticamente significativas y positivas, convendría destacar los datos obtenidos entre el compromiso afectivo y claridad en los sentimientos $(r=.32)$. De igual forma, resulta necesario despuntar la puntuación que se desprende en el par compromiso normativo y claridad en los sentimientos $(r=.25)$.

\section{Discusión}

El propósito central de esta investigación se centró en conocer el papel que ejercen el compromiso organizacional y la inteligencia emocional en el perfil de los mediadores del Poder Judicial de Oaxaca. Pues bien, de manera general, con base en los resultados obtenidos, podríamos avanzar que este objetivo se ha cubierto. Aún más, podríamos decir que tanto el compromiso como la inteligencia emocional son constructos fundamentales para definir el perfil del mediador en México.

De modo más específico, y atendiendo a los postulados establecidos en las hipótesis de trabajo, convendría decir que la primera de ellas, donde pronosticábamos que los tres aspectos del compromiso (afectivo, normativo y calculado) ejercerán notables influencias en el sexo de los participantes, siendo la vertiente normativa la que desempeña un papel central, se cumple. En este sentido, los datos indican que el compromiso normativo, es decir, la creencia en la lealtad de la organización, en un sentido moral, de alguna manera como pago, quizá por recibir ciertas prestaciones, tienen cierta influencia entre el sexo de los participantes. Luego con estos datos podríamos decir que se desarrolla un fuerte sentimiento de permanecer en la institución, como efecto de experimentar una sensación de deuda hacia la organización por haberle dado la oportunidad o recompensa que fue valorada por el trabajador, siendo, con toda probabilidad, diferente entre hombres y mujeres. 
En lo que respecta a la segunda hipótesis, donde pronosticábamos que los tres componentes del compromiso (afectivo, normativo y calculado) influirán de manera significativa en la edad de los participantes, mostrándose puntuaciones superiores en la vertiente afectiva, debemos decir que, también, se corrobora. En este sentido, con estos datos podemos afirmar que la vinculación emocional o, lo que es lo mismo, el apego afectivo, es un aspecto importante según la edad de los participantes. Estos resultados se encuentran en línea con los datos obtenidos por diversos autores (Salvador, 2005; Salvador \& Morales, 2009). En concreto, según estos autores para conseguir la estabilidad institucional, resulta imprescindible potenciar esta vertiente del compromiso. De acuerdo con esta forma de proceder, conseguiremos que los trabajadores se sientan orgullosos de pertenecer a la organización.

Cuando nos detenemos en la tercera hipótesis, donde estimamos que los tres componentes de la inteligencia emocional (atención y percepción emocional, claridad de los sentimientos y manejo de las emociones) presentan notables repercusiones en la edad de la muestra, siendo ligeramente superior el manejo de las emociones, tenemos que decir que se cumple de manera parcial. En concreto, nuestros datos apuntan a que efectivamente los tres componentes de la inteligencia emocional repercuten en la edad, aunque es la vertiente claridad de los sentimientos la que presenta una mayor repercusión. Estos resultados podrían hacernos pensar que en la medida en que las personas aumentan su edad tienen más claros sus sentimientos, lo que, a priori, los hace más competentes para el dominio de sus emociones.

En lo concerniente a la cuarta hipótesis, donde consideramos que los componentes de la inteligencia emocional (atención y percepción emocional, claridad de los sentimientos y manejo de las emociones) se relacionan fuertemente con la edad, mostrándose elevada la relación entre atención y percepción, se confirma. Por ende, todo apunta a que existen diferencias por género en la forma de atender y percibir las emociones. Podríamos decir que estos hallazgos coinciden con los obtenidos por Salvador (2009) en una mues- tra mexicana. Según esta autora, este aspecto es fundamental, especialmente, cuando se trata de emitir actuaciones para emprender.

En último lugar, en lo que respecta a las hipótesis quinta -donde esperábamos que existiera una relación estadísticamente significativa y negativa entre el compromiso afectivo y la atención y percepción emocional- y la hipótesis sexta, según la cual estimábamos que existiera una fuerte relación, es decir, estadísticamente significativa y positiva, entre el compromiso afectivo y la claridad de los sentimientos, podemos corroborar el cumplimiento de las mismas. Estos datos parecen indicar que cuando mayor es el vínculo emocional menor es la atención y percepción de las emociones. Se trata, pues, de que al desarrollar un mayor vínculo afectivo parece que se pierde el control de las emociones porque se desarrolla una mayor empatía. Por su parte, el desarrollo del compromiso afectivo se relaciona positivamente con la claridad de los sentimientos, es decir, es posible establecer esta empatía porque tenemos claros los sentimientos de los demás. Así mismo, los datos que se desprenden de la Figura 5 nos indican, no sólo lo que acabamos de exponer, sino, también, que el compromiso normativo se vincula positivamente con la claridad de los sentimientos. En línea con esto, para que se desarrolle un sentimiento de lealtad hacia la institución, resulta imprescindible tener claros los sentimientos.

Los datos expuestos anteriormente adquieren sentido si tenemos en cuenta el perfil de la muestra, es decir, mediadores mexicanos. Estas personas, para poder ejercer a la perfección su rol necesitan ser neutrales, esto es, no implicarse emocionalmente en su tarea. Por tanto, parece que el compromiso afectivo, dentro de este contexto laboral quedará en segundo lugar. Así, a priori, parece que los datos se alejan bastante de los obtenidos en otros estudios (Salvador, 2005). No obstante, esto no es así, dado que según reflejan los mediadores, resulta imprescindible desarrollar una vinculación de tipo normativo (ver resultado de la Figuras 1 y 2). Este hecho indica que los mediadores desarrollan un sentimiento de lealtad en la organización, o sea, en un sentido moral, que varía según la edad y el sexo, pero 
que en ambos casos se desarrolla. Podríamos decir que no ejercen vínculos emocionales porque precisamente en su puesto de trabajo esta forma de proceder no resulta ser la más eficaz, aunque se encuentran muy comprometidos con su institución. Con todo, con este tipo de compromiso se desarrolla un fuerte sentimiento de permanecer en la institución, como efecto de experimentar una sensación de deuda hacia la organización por haberle dado la oportunidad o recompensa que fue valorada por el trabajador.

De igual forma, quisiéramos resaltar que para los mediadores mexicanos la inteligencia emocional es otro factor importante para el éxito laboral, existiendo variaciones por edad y sexo. Dentro de este punto, debemos subrayar que parecen existir dos dimensiones relevantes, siendo un poco más precisos, podríamos mencionar la atención y percepción emocional $y$, también, la claridad en los sentimientos. En este sentido, convendría distinguir que la percepción de los mediadores parece estar centrada tanto en comprender sus propias emociones como la de los demás. Ahora bien, si tenemos presente los datos obtenidos en la última Figura (5), podríamos entender que sea el compromiso normativo el que se vincule fuertemente con la claridad de los sentimientos de los demás.

En suma, para ser un buen mediador en México, los datos parecen apuntar a que deben estar comprometidos normativamente, es decir, desarrollar lealtad a la institución, mostrar vínculo emocional, aunque éste muestra una relación baja y positiva con la atención y percepción de sus sentimientos, dado que de no ser así entorpecería el desarrollo exitoso de su puesto. Además, conviene subrayar que el vínculo emocional resulta fundamental para tener claros los sentimientos de los demás. A todo esto, debemos añadir que, tanto en lo que respecta al compromiso como en lo concerniente a la inteligencia emocional, existen diferencias significativas según la edad y el sexo de los participantes.

No obstante, dado su carácter exploratorio, los resultados de esta investigación deben ser tomados con cautela, ya que nuestro trabajo no está exento de limitaciones. Podríamos destacar que, probablemente, la más importante se encuentra inherente al propio estudio, provocada sobre todo por el tamaño muestral. Otro inconveniente reside en el momento de llevar a cabo la recogida de datos, lo que queremos decir exactamente es que los resultados se obtuvieron en un momento temporal único, lo que podría interferir en las relaciones encontradas entre las variables. Además, creemos necesario profundizar en el análisis del sentimiento de lealtad de los mediadores mexicanos, en concreto, resulta necesario averiguar si esta forma de proceder está marcada bien por factores culturales 0 , contrariamente, por aspectos familiares (Morrow, 1983). Por todo ello, enfatizamos la necesidad de continuar trabajando dentro de esta línea, con el principal propósito de ahondar en las causas y la idiosincrasia del perfil del mediador mexicano.

\section{Referencias}

Arciniega, L.M. (2002). Compromiso Organizacional en México: ¿Cómo hacer que la gente se ponga la camiseta? Dirección Estratégica. Revista de Negocios del ITAM, 2(1), 21-23.

Allen, N.J. \& Meyer, J.P. (1990). The measurement and antecedents of affective, continuance and normative commitment to the organization. Journal of occupational, 63 (1), 1-18.

Becker, H.S. (1960). Notes on the concept of commitment. American Journal of Sociology, 66, 350-360.

Buchanan, B. (1974). Building organization commitment: The socialization of managers in workers organizations. Administrative Science Quarterly, 19, 533-546.

Cohen, A. y Lowenberg, G. (1990). A re-examination of the Side-Bet theory as applied to Organizational Commitment: a meta-analysis. Human Relations, 43 (10), 1015-1050.

Cotton, J.L. (1993). Employee involvement. Nueva York: Sage. 
Cook, K. S. y Emerson, R. M. (1978). Power, equity and commitment in exchange networks. American Sociological Review, 43 (5), 721739.

Churchill, G.A. (1979). A paradigm for developing better measures of marketing construcs. Journal of Marketing Research, 16 (1), 64-73.

Eisenberger, R., Hungtington, R., Hutchison, S. \& Sowa, D. (1986). Perceived Organizational Support. Journal of Applied Psychology, 71, 500-507.

Etzioni, A. (1975). A comparative analysis of complex organizations. Nueva York: Free Press.

Franklin, J.L. (1975). Power and commitment-empirical assessment. Human Relations, 28 (8), 737-753.

Goleman, D. (1998). Working with emotional intelligence. Nueva York: Bantam Books.

Goleman, D. (2001). Emotional intelligence: Perspectives on a theory of performance. En C. Cherniss y D. Goleman (Eds.), The emotionally intelligent workplace. (24-30). San Francisco: Jossey-Bass.

Guest, D. (1995). Human resource management, trade unions and industrial relations. En J. Storey (Ed.), Human resource management. A critical text. London: Routledge.

Kanter, R.M. (1968). Commitment and social organization. A study of commitment mechanisms in utopian commumities. American Sociologial Review, 33, 499-517.

Lyons, S.T., Duxbury, L.E. \& Higgins, C.A. (2006). A Comparison of the Values and Commitment of Private Sector, Public Sector, and Parapublic Sector Employees. Public Administration Review, 66 (4), 605-18.

Mathieu, J.E. \& Zajac, D. (1990). A review and meta-analysis of the antecedents, correlates, and consequences of organizational commitment. Psychological Bulletin, 108, 171-194.

Meyer, J.P. \& Allen, N.J. (1997). Commitment in the workplace: Theory, research and application. Thousand Oaks: C. A.: Sage.

Meyer, J.P. \& Allen, N.J. (1984). Testing the side best theory of organization commitment: some methodological considerations. Journal of Applied Psychology, 69, 372-378.

Meyer, J.P., Stanley, D.J., Herscovitch, L. \& Topolnytsky, L. (2002). Affective, Continuance, and Normative Commitment to the Organization: A Meta-analysis of Antecedents, Correlates, and Consequences. Journal of Vocational Behavior, 61, 20-52.

Morgan, R. M. y Hunt, S. D. (1994). The commitment trust theory of relationship marketing. Journal of Marketing, 58 (3), 20-38.

Morrow, P.C. (1983). Concept redundancy in organizational research: The case of work commitment. Academy of Management Review, 8, 486-500. Mowday, R.T., Steers, R.M. y Porter, L.W. (1979). The measurement of organizational commitment. Journal of Organizational Behavior, 16, 224-247.

Meyer, J. P., Allen, N.J. \& Smith, C.A. (1993). Commitment to organizations and occupations: Extension and test of a three-component conceptualization. Journal of Applied Psychology, 78 (4), 538-551.

Mowday, R.T., R. Steers, \& Porter. L. (1979). The measurement of organizational commitment. Journal of Vocational Behavior, 14, 224-47.

O’Really, C. \& Chatman, J. (1986). Organizational commitment and psychological attachment: The effects of compliance, identification, and internalization on prosocial behavior. Journal of Applied Psychology, 71, 492-499.

Peter, J.P. (1979). Reliability: A review of psychometric basics and recent marketing prac- 
tices. Journal of Marketing Research, 16 (1), 6-17.

Porter, L.W. \& Lawler, E.E. (1965). Properties of organization structure in relation to job attitudes and job behavior. Psychological Bulletin, 64, 23-51.

Reichers, A. E. (1986). Conflict and organizational commitment. Journal of Applied Psychology, 71, 508-514.

Rego, A. \& Fernández, C. (2005). Inteligencia emocional: Desarrollo y validación de un instrumento de medida. Revista Interamericana de Psicología, 1, 23-28.

Robbins, S. P. (1999). Comportamiento Organizacional. México: Prentice-Hall Salancik, G. R. (1977). Commitment and control organizational behavior and belief. En B. M. Staw G. R. Salancik (Eds.). (pp.19-38). New Directions in Organizational Behaviour. Chicago: St Clair Press.

Salovey, P. \& Mayer, J.D. (1990). Emotional intelligence. Imagination, cognition and personality, 9, 185-211.

Salvador, C. (2005). Validez predictiva de los componentes del compromiso del cliente y su relación con la satisfacción. Anales de Psicología, 21 (2), 316-322.

Salvador, C. (2008a). Impacto de la inteligencia emocional percibida en el autoempleo. Boletín de Psicología, 92, 65-80.

Salvador, C. (2008b). Calidad de servicios: El camino hacia la fidelidad del consumidor. Universidad de Almería: Servicio de Publicaciones.

Salvador, C. (2009). Ecuaciones estructurales como modelos predictivos de la autoeficacia emprendedora en una muestra de jóvenes mexicanos y españoles. Apuntes de Psicología, 27 (1), 65-78.

Salvador, C. \& Mañas, M.A. (2009). Actitudes hacia el trabajo: satisfacción, implicación y compromiso. En Rodríguez, A., Zarco, V. \& González, J.M. (Eds.). Psicología del Trabajo. Madrid: Pirámide.

Salvador, C. \& Morales, J.M. (2009). Satisfacción Vital e Inteligencia Emocional como variables moduladoras de la Autoeficacia emprendedora de los jóvenes mexicanos. Revista Interamericana de Psicología, 43 (2), 267-277. 
